\title{
Gastrointestinal permeability changes in the preterm neonate
}

\author{
RICHARD C BEACH, IAN S MENZIES, GRAHAM S CLAYDEN, AND JON W SCOPES
}

Department of Paediatrics and Department of Chemical Pathology, St Thomas's Hospital Medical School, London

SUMMARY The lactulose/L-rhamnose urinary excretion ratio during continued infusion of milks containing both sugars was used as an index of the permeability of the neonatal bowel to large and small molecules. Healthy infants of gestational age 31-36 weeks proved to have a period of enhanced permeability to lactulose during the first week of life, the lactulose/L-rhamnose excretion ratio being significantly higher on day 2 than on days 9 or 16 when a mature pattern of permeability could be seen. In infants traumatised by asphyxia or sepsis this change was much less pronounced. Healthy preterm infants of gestational age 26-29 weeks showed a 'mature' pattern of permeability at birth, followed by a temporary period of enhanced permeability after 3-4 weeks of life. It is proposed that enhanced permeability to larger molecules is a specific temporary condition of the neonatal bowel in man as in other mammals, but the immunological implications in man remain to be established.

The lining of the adult gastrointestinal tract acts as a barrier restricting the passage of large molecules. In neonatal mammals gastrointestinal permeability to large molecules is known to be enhanced and intact immunoglobulins from maternal milk may be absorbed by pinocytosis. ${ }^{1}$ The term 'gut closure' describes the subsequent reduction in permeability to an 'adult' pattern. In the human neonate immunoglobulins are largely acquired transplacentally and the role of the bowel has not been fully elucidated. Although there is evidence that intact proteins and other macromolecules may permeate the bowel both in healthy infants and adults, ${ }^{23}$ proof that this transfer is enhanced during the human neonatal period is inconclusive. Thus, although antibodies to food allergens are more in evidence during early infancy than later in life, ${ }^{4}$ this could equally represent an alteration of immune response or of antigen permeation. Similarly, the pronounced lactosuria during the first week of life described by Bickel $^{5}$ might be the result either of a transient increase in gastrointestinal permeability or of a temporary lactase deficiency. The disaccharide lactulose which resists the action of human intestinal hydrolases $^{6}$ appears to provide a means of distinguishing these possibilities. ${ }^{7}$

The state of gastrointestinal permeability may be expressed by comparing the absorption of markers of different sizes. ${ }^{8} 9$ As part of a study of gastro- intestinal function in preterm infants a method for assessing gastrointestinal permeability using lactulose and L-rhamnose was developed for use in human neonates.

\section{Methods}

Lactulose content of infant feeds. Liquid infant milk formulae supplied to hospitals contain lactulose, ${ }^{10}$ which is formed from lactose during heat sterilisation. A reliable quantitative paper chromatographic method has been developed which shows the mean lactulose content of the Premium (Cow \& Gate) milk in use at this hospital to be $13.1 \mathrm{mmol} / 1$.

Protocol for permeability testing in preterm infants using sugars as markers. Test milks were prepared by adding lactulose or L-rhamnose, or both, to Premium (Cow \& Gate) or to expressed breast milk to yield a final concentration of lactulose 13.1 $\mathrm{mmol} / \mathrm{l}$ and L-rhamnose $3.0 \mathrm{mmol} / \mathrm{l}$ in the milk. Infants were then fed on this test milk for periods of 24-36 hours. The type, timing, and volume of feeds varied with each infant's age, weight, and clinical condition, and no changes were made for the purposes of this study. Urinary excretion of the marker sugars was assessed either from a 12-hour urine sample collected between the 24th and 36th hour of the test, or from random urine samples 
collected after 24 hours. Urine samples were preserved with thiomersalate (minimum $10 \mathrm{mg}$ / $100 \mathrm{ml}$ urine ${ }^{11}$ ) and stored at $-20^{\circ} \mathrm{C}$ for analysis. Lactulose was analysed by quantitative paper chromatography, and L-rhamnose by quantitative thin-layer chromatography. ${ }^{9}$

\section{Patients.}

\section{Group 1}

The 29 healthy preterm infants in this group had a mean gestational age (according to Dubowitz et $a l .{ }^{12}$ ) of 34 weeks (range 31-36) and a mean weight of $1721 \mathrm{~g}$ (1150-2290). All had Apgar scores greater than 5 at one minute and 10 at ten minutes. None had suffered respiratory distress or required intravenous therapy, and all were asymptomatic and on full oral feeding by the second day of life when the first test was carried out. If possible further tests were performed on the 9th and 16th days.

\section{Group 2}

The 7 infants in this group had a mean gestational age of 27.5 weeks (range 26-29) and a mean weight of $1040 \mathrm{~g}$ (range 870-1500), and all fulfilled the Apgar criteria described above. Two of these infants suffered recurrent apnoea during the first 3 weeks of life and four had intravenous fluids. All had started oral feeding by the 4th day of life. Tests were carried out at weekly intervals if possible.

\section{Group 3}

The 5 infants in this group had all suffered severe perinatal insults. Their mean gestational age was 37.5 weeks (range 33-40) and their mean weight was $3140 \mathrm{~g}$ (range 1390-5300). Three of these infants suffered severe birth asphyxia with Apgar scores less than 5 at ten minutes. One developed streptococcal meningitis on the second day of life and one suffered severe polycythaemia and hypoglycaemia.

An additional control group of 30 healthy children and adults was studied. After an overnight fast they each took an isotonic oral load containing lactulose $5 \mathrm{~g}$ and L-rhamnose $1 \mathrm{~g}$. Urinary excretion of lactulose and L-rhamnose was determined from a subsequent 5-hour urine collection.

All these studies were approved by the hospital ethical committee.

Bacterial decomposition of sugar markers. The possibility that bacterial degradation of marker sugars might invalidate these test procedures was investigated by deliberately contaminating test milks containing lactulose and L-rhamnose with samples of the upper intestinal flora from preterm neonates and incubating the mixture in vitro at $37^{\circ} \mathrm{C}$ under aerobic and microaerophyllic conditions. Only slight changes in the ratio of lactulose to L-rhamnose were detected in any sample of milk during the first 4 hours of incubation although there was evidence of lactose hydrolysis.

\section{Results}

Sugar ratios were compared in pairs of random urine samples collected between the 24th and 36th hours of test milk feeding in 13 infants from group 1. No significant differences were found between these early and late urine samples, indicating that a steady state of intestinal sugar permeation and excretion had been achieved by the 24th hour of oral test milk administration. Such a 'steady state' implies that the rate of renal excretion of the sugar markers, both of which resist metabolism, is equivalent to the rate of permeation from the intestinal lumen. Accurate 12-hour urine collections were made from 13 infants in group 1, and the percentage absorption of lactulose and L-rhamnose calculated by comparing the hourly urinary output with the oral input. Percentage L-rhamnose ab8 sorption, which varied between 12 and $40 \%$ (average $25 \%$ ) of oral intake, showed no significan difference between the 2 nd and 9 th days. In contrast? lactulose absorption was significantly higher on the 2nd day of life when the average absorption was $3 \cdot 2 \%$ (range $1 \cdot 2-7 \cdot 0$ ) compared with 9th day when the average absorption was $1.2 \%$ (range $0 \cdot 7-1.9$ ) $(P<\mathbf{0 . 0 1}$, Wilcoxon's ranked sum test).

The state of gastrointestinal permeability is better expressed as the lactulose/L-rhamnose excretion ratio, which may be calculated using data from urine collections or from random urine samples as follows:

$\%$ oral lactulose excreted $=$

Ulactulose $\times$ UV $/$ M lactulose $\times$ MV $(\times 100)$

$\%$ oral rhamnose excreted $=$

U rhamnose $\times \mathrm{UV} / \mathrm{M}$ rhamnose $\times \mathrm{MV}(\times 100)$

$\%$ lactulose excretion $/ \%$ rhamnose excretion $=$

U lactulose $\times$ M rhamnose/U rhamnose $\times M$ lactulose $=$ lactulose/L-rhamnose excretion ratio.

Where $\mathrm{U}$ lactulose $=$ urine lactulose concentration; $\mathrm{U}$ rhamnose = urine L-rhamnose concentration; M lactulose $=$ test milk lactulose concentration; $\mathbf{M}$ rhamnose = test milk L-rhamnose concentration; $\mathrm{MV}=$ volume of milk given per hour; $\mathrm{UV}=$ volume of urine passed per hour. 
The infants in group 1 had a significantly higher lactulose/L-rhamnose excretion ratio on day 2 than on day 9 (Fig. 1) indicating an increased gastrointestinal permeability to larger molecules in the first week of life. No explanation was apparent for the very high lactulose/L-rhamnose excretion ratio in 5 infants from group 1 (Fig. 1) although they appear to form a separate group. These infants have been included in the non-parametric statistical analysis. There was no significant change in permeability between the 9th and 16th day in this group. The extremely preterm infants in group 2 do have a period of increased permeability with high lactulose/ L-rhamnose excretion ratio, but it occurs later in life when the infants are 3-4 weeks old (Fig. 2). In this group values for the lactulose/L-rhamnose excretion ratio are significantly higher during the 3rd or 4th weeks of life than values obtained either earlier or later.

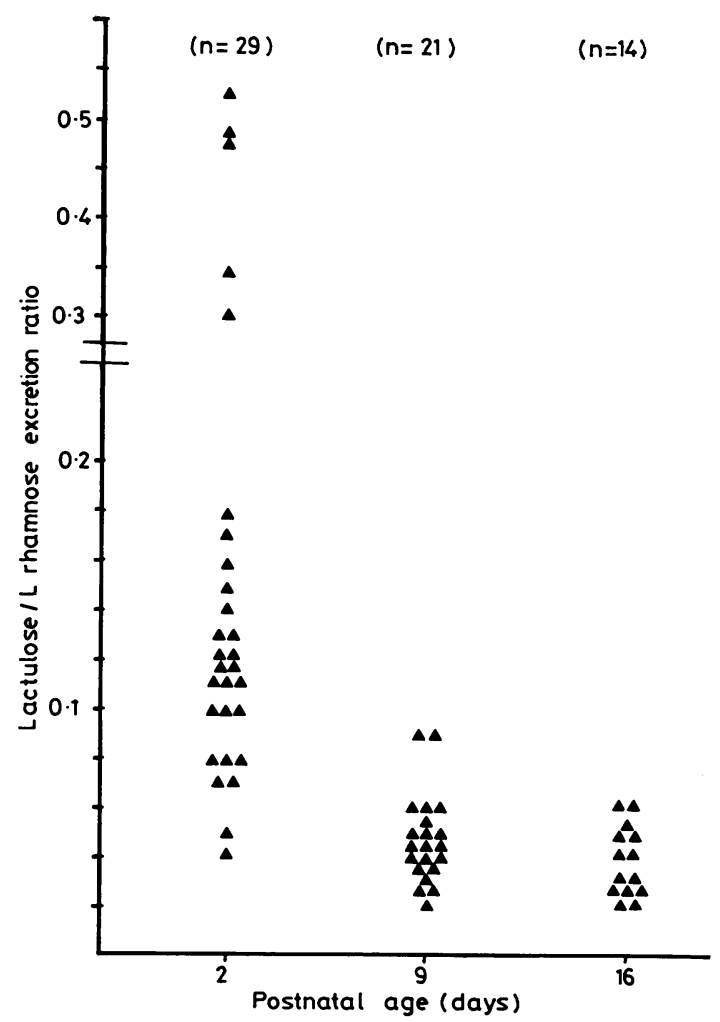

Fig. 1 Lactulose/L-rhamnose percentage urinary excretion ratios during continued test milk infusion in healthy preterm infants of 31-36 weeks' gestational age (group 1).

Statistical analysis (Wilcoxon's ranked sum test): Day 2 v day 9: $P<0 \cdot 01$.

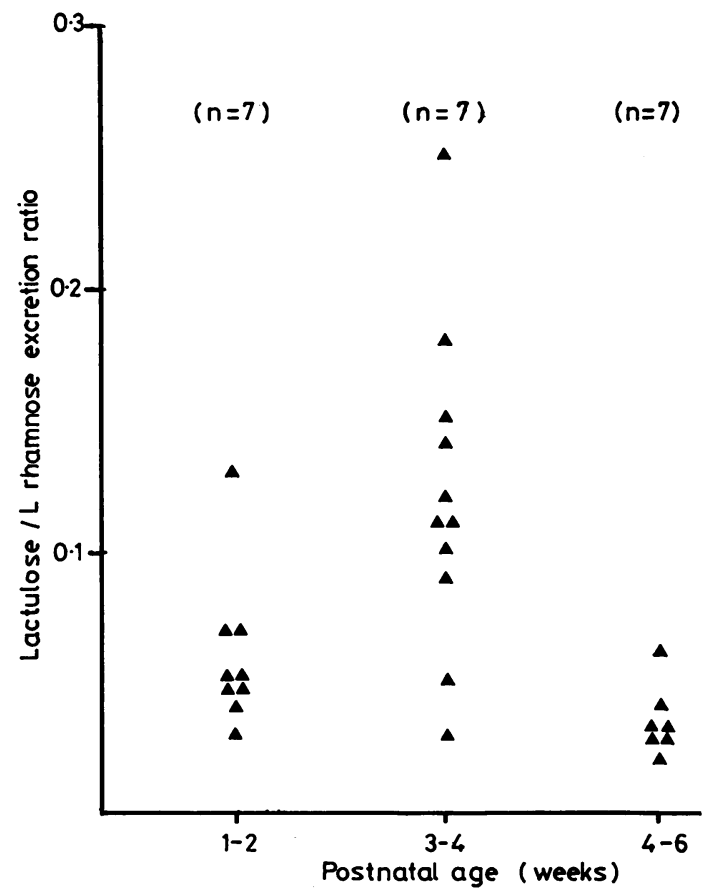

Fig. 2 Lactulose/L-rhamnose percentage urinary excretion ratios during continued test milk infusion in healthy preterm infants of 26-29 weeks' gestational age (group 2).

Statistical analysis (Wilcoxon's ranked sum test):

Week 1-2 v week 3-4: $P<0.02$; week 5-6 v week 3-4: $P<0.02$.

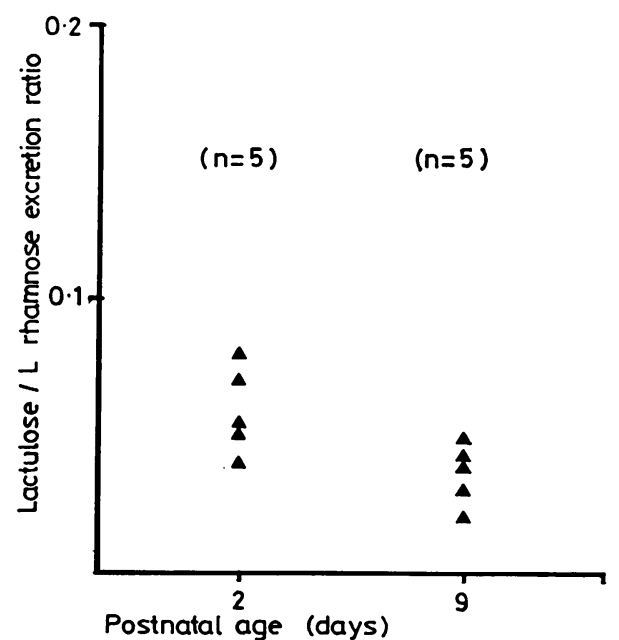

Fig. 3 Lactulose/L-rhamnose percentage urinary excretion ratios during continued test milk infusion in septic or asphyxiated infants (group 3). 
The damaged infants in group 3 have a higher lactulose/L-rhamnose excretion ratio on day 2 than on day 9 (Fig. 3), but the extent of this increased permeability on day 2 is significantly less than that observed in the healthy infants from group 1 . The healthy children and adults had a lactulose/Lrhamnose excretion ratio of $0 \cdot 04 \pm 0.02$.

\section{Discussion}

The lactulose/L-rhamnose urinary excretion ratio after simultaneous ingestion of these two sugars was used as an index of the permeability of the bowel to large and small molecules. The suitability of these two sugars, which pass across the intestinal wall by unmediated diffusion and are fully excreted into the urine after reaching the blood stream, for assessing intestinal permeability by this indirect method, has already been established. ${ }^{6}{ }^{9}$ As has been shown, these sugars also resist degradation by upper intestinal flora when added to infant feeds.

The percentage of L-rhamnose absorbed after oral administration of isotonic test solutions is 20 to 30 times greater than that of lactulose. Thus healthy children and adults demonstrate a lactulose/Lrhamnose percentage urinary excretion ratio of $0.04 \pm 0.02$, to be regarded as the 'mature' pattern of intestinal permeability. This difference between the absorption of lactulose and L-rhamnose is much greater than would be expected for unmediated diffusion on the basis of molecular weight (342 and 164 Daltons respectively) unless restriction related to pore size is assumed. At least two pathways of unmediated permeation for polar molecules have been suggested, one with high capacity accommodating small molecules (180 Daltons and below), ${ }^{13}$ through which L-rhamnose, but not lactulose, could pass, and the other of low capacity capable of accommodating larger molecules including lactulose. These pathways have been ascribed to a population of small water-filled 'pores'14 of high incidence probably situated in cell walls, and a much lower incidence of larger channels thought in mature bowel to be chiefly paracellular and possibly associated with tight junctions. ${ }^{15-17}$ Changes in the capacity of these two routes of permeation caused, for example, by neonatal pinocytosis or disruption of the intracellular tight junctions would be reflected in the relative absorption of lactulose and Lrhamnose, conveniently expressed as the percentage urinary lactulose/L-rhamnose ratio after simultaneous oral administration of both sugars as a mixture. The lactulose molecule is very much smaller than most biological macromolecules. However, once a critical size has been exceeded, there is evidence that similar permeability patterns may be demonstrated using polysaccharides of $\Omega$ molecular weight up to $3000 .^{15}$

Combination of both permeability markers in the same test system offers some important advantages. $\overrightarrow{\vec{F}}$ The effects of irrelevant variables-such as gastric $\frac{\infty}{7}$ emptying, dilution by intestinal fluid, transit rate, volume distribution within body spaces, renal $\frac{\partial}{\omega}$ clearance, and reliability of urine collection-are $\frac{\bar{\phi}}{\bar{\phi}}$ conveniently avoided. Although these factors may $\unrhd$ alter total recovery, they make little difference to the \& urinary excretion ratio which is determined solely by $\overrightarrow{0}$ the state of intestinal permeability.

The bolus oral loading technique suitable for $\overrightarrow{\vec{\omega}}$ children and adults ${ }^{9}$ was inappropriate for neonates $\frac{\Omega}{\Delta}$ to whom a fasting period followed by administration of an aqueous solution of test sugars would be or disturbing. In place of this a continuous test feeding $N$ system has been substituted, urine samples being obtained after 24 hours when a steady state had $\vec{\nexists}$ been established. This test system causes little 응 disturbance to infants and can be initiated without preparatory fasting. Furthermore marker sugar 71 excretion depends mainly on absorption at this $\frac{\mathbb{C}}{0}$ steady state and is largely independent of possible changes in renal function, as alterations in renal sugar clearance are balanced by changes in plasma $\overrightarrow{0}$ sugar concentration. The markers are considereg harmless at the dose levels used: lactulose is well-known constituent of infant milk feeds ofter present at concentrations similar to those employe and L-rhamnose a naturally-occurring sugar without $\overline{0}$ known biological effects. Further alterations in the $\stackrel{0}{\approx}$ normal feeding regimen are not required, and only $\stackrel{\square}{\square}$ random urine samples need be collected.

The patterns of intestinal permeability obtained from infants in the present study show interesting differences from those found in children and adults. Urinary lactulose/L-rhamnose excretion ratios obtained from preterm neonates (group 1) on the second day of life are raised in comparison with $\frac{0}{7}$ ratios measured on day 9 and with adult ratios. That this is the result of an increase in the perme- $\delta$ ability of the bowel to lactulose rather than of decreased permeation of L-rhamnose is confirmed by $\mathrm{O}$ data from those infants in group 1 who had timed urine collections in whom lactulose excretion was increased on day 2, L-rhamnose remaining unchanged between days 2 and 9 .

This early increase in permeability to lactulose $N$ could theoretically result from impairment of the N intestinal mucosal integrity associated either with 'immaturity' or with the traumas of birth. However, the infants from group 2 maintain a mature pattern for between 1 and 2 weeks after delivery, despite extreme prematurity. Their period of enhanced permeability is not manifest until 3-4 weeks of age. . 
The septic or asphyxiated infants from group 3 do have an early period of enhanced permeability, but this is less pronounced than that found in the normal preterm infants (group 1). There is therefore good reason to propose that this temporary period of enhanced intestinal permeability is a specific condition of the early neonatal period not generally manifest until the bowel is sufficiently mature, and further that it is inhibited rather than accentuated by severe birth asphyxia or sepsis. Early results using a suitable modification of this method in term infants appear to indicate a similar period of enhanced permeability early in neonatal life.

It is not certain that molecules as large as proteins share this neonatal enhanced transfer and interactions of immunological significance between human neonatal bowel and maternal colostrum remain to be established. However, we have shown that the bowel of the preterm neonate undergoes permeability changes similar to those demonstrated in neonatal mammals. Could it be that this period of enhanced permeability has some functional significance in the colostrum-fed infants, or does it represent a vestigeal remnant of gastrointestinal development in lower mammals?

We thank the Sister and staff of the Special Care Baby Unit for invaluable help, and Mrs Gale Willner for secretarial assistance.

St Thomas's Hospital Endowment Fund provided financial support.

\section{References}

1 Walker W A. Gastro-intestinal host defence: importance of gut closure in control of macromolecular transport. In: Elliott K M, Whelan J, eds. Development of mammalian absorptive processes. CIBA Foundation Symposium No 70 (New Series). Amsterdam: Exerpta Medica, 1979: 201-19.

2 Anonymous. Antigen absorption by the gut. Lancet 1978; ii: 715-7.

3 Walker W A, Isselbacher K J. Uptake and transport of macromolecules by the intestine: possible role in clinical disorders. Gastroenterology 1974; 67: 531-50.

4 Eastham E J, Lichauco T, Grady M I, Walker W A. Antigenicity of infant formulas. Role of immature intestine on protein permeability. $J$ Pediatr 1978; 93: $561-4$.

5 Bickel H. Mellituria, a paper chromatographic study. J Pediatr 1961 ; 59: 641-56.

6 Dahlqvist A, Gryboski J D. Inability of the human small intestinal lactase to hydrolyze lactulose. Biochim Biophys Acta 1965; 110: 635-6.

7 Müller M, Walker-Smith J, Shmerling D H, Curtius H C, Prader A. Lactulose: a gas liquid chromatography method of determination and evaluation of its use to assess intestinal mucosal damage. Clin Chim Acta 1969; 24: 45-9.

8 Chadwick V S, Phillips S F, Hofmann A F. Measurements of intestinal permeability using low molecular weight polyethylene glycols (PEG 400). I. Chemical analysis and biological properties of PEG 400 . Gastroenterology 1977; 73: 241-6.

9 Menzies I S, Laker M F, Pounder R, et al. Abnormal intestinal permeability to sugars in villous atrophy. Lancet 1979; ii: 1107-9.

10 Bernhart F W, Gagliardi E D, Tomarelli R M, Stribley R C. Lactulose in modified milk products for infant nutrition. J Dairy Sci 1964; 48: 399-400.

11 Menzies I S. Quantitative estimation of sugars in the blood and urine by paper chromatography using direct densitometry.J Chromatogr 1973; 81 : 109-27.

12 Dubowitz L, Dubowitz V, Goldberg C. Clinical assessment of gestational age in the newborn infant. $J$ Pediatr $1970 ; 77: 1-10$.

13 Höber R, Höber J. Experiments on the absorption of organic solutes in the small intestine of rats. $J$ Cell Comp Physiol 1937; 10: 401-22.

14 Fordtran J S, Rector F C, Jr, Ewton M F, Soter N, Kinney J. Permeability characteristics of the human small intestine. J Clin Invest 1965 ; 44: 1935-44.

15 Wheeler P G, Menzies I S, Creamer B. Effect of hyperosmolar stimuli and coeliac disease on the permeability of the human gastrointestinal tract. Clin Sci Mol Med $1978 ; 54: 495-501$.

16 Loehry C A, Axon A T R, Hilton P J, Hider R C, Creamer B. Permeability of the small intestine to substances of different molecular weight. Gut 1970; 11: 466-70.

17 Smulders A P, Wright E M. The magnitude of nonelectrolyte selectivity in the gallbladder epithelium. J Membr Biol 1971 ; 5: 297-318.

Correspondence to Dr R C Beach, Department of Paediatrics, Southampton General Hospital, Tremona Road, Southampton SO9 4XY.

Received 30 March 1981 